\title{
Rotational kinematics influence multimodal perception of heaviness
}

\author{
Matthew StReit and KeVin Shockley \\ University of Cincinnati, Cincinnati, Ohio \\ ANTHONY W. Morris \\ University of Alabama, Huntsville, Alabama \\ AND \\ Michael A. RiLey \\ University of Cincinnati, Cincinnati, Ohio
}

\begin{abstract}
Perceived heaviness has been shown to be specific to an object's rotational inertia $(I)$, its resistance to rotational acceleration. According to the kinematic specification of dynamics (KSD) principle, we hypothesized that $I$ is optically specified by rotational kinematics. Using virtual depictions of wielded objects, we investigated whether the visually detected rotational kinematics of wielded objects would influence perceived heaviness in a manner consistent with the inertial model of heaviness perception. We scaled the virtual object's movement so that it rotated more or less than its wielded counterpart, specifying lower and higher $I$, respectively. Perceived heaviness was inversely related to the rotational scaling factor, consistent with a KSD interpretation of the inertial model.
\end{abstract}

The absence of a direct correspondence between changes in object mass and perceived heaviness has been recognized since Weber's (1834/1978) original psychophysical investigations. The mismatch between perceived heaviness and object mass is, perhaps, most readily illustrated by the classic size-weight (S-W) illusion (Charpentier, 1891). A wielded object of a given mass generally feels lighter as object volume increases. This phenomenon has been replicated many times (e.g., Ellis \& Lederman, 1993; Stevens \& Rubin, 1970) and may reflect perceptual attunement to the pattern of the object's resistances to rotational accelerations - its rotational inertia - detected during wielding (Amazeen \& Turvey, 1996; Shockley, Carello, \& Turvey, 2004; Shockley, Grocki, Carello, \& Turvey, 2001; Turvey, Shockley, \& Carello, 1999).

In its simplest characterization, rotational inertia corresponds to the mass of an object multiplied by the distance squared of the center of mass from the point of rotation (e.g., the wrist). Generally speaking, the lower the overall rotational inertia, the lighter an object feels (Amazeen \& Turvey, 1996, Experiment 1). For example, a hammer grasped at the base of the handle has greater rotational inertia (i.e., it takes more force to wield it) than when grasped at the head. When grasped at the handle most of the mass is far away from the wrist, but when grasped at the head, most of the mass is near the wrist. The hammer will, accordingly, feel heavier when grasped by the handle than when grasped at the head. Amazeen and Turvey rec- ognized that as the S-W illusion stimuli used by Stevens and Rubin (1970) increased in volume, the objects' rotational inertia along the three spatial dimensions became more similar. Amazeen and Turvey replicated those inertial patterns with no corresponding change in volume and found the same change in heaviness as that observed by Stevens and Rubin. The implication is that heaviness perception reflects perceptual sensitivity to the inertial properties of wielded objects.

It has also been demonstrated, however, that heaviness perception is additionally influenced by vision. Masin and Crestoni (1988) and Ellis and Lederman (1993) showed that objects of a given mass lifted by a string (i.e., not grasped and wielded) were perceived as lighter as the objects increased in size. Amazeen (1997) manipulated object size while controlling for rotational inertia and found that perceptual reports obtained in the absence of vision conformed qualitatively to Amazeen and Turvey's (1996) inertial model. When vision was allowed, however, objects consistently felt lighter with increasing volume, although the manner of change in volume (width vs. length) influenced heaviness differently. The latter finding suggests that volume, per se, was not the source of the influence. Masin and Crestoni likewise found that the influence of vision cannot be attributed solely to object size. They manipulated when participants could see the objects by allowing the participants to look just prior to, just after, or during lifting. They found an influence of

K. Shockley, kevin.shockley@uc.edu 
vision only when the participants looked while lifting the objects. The implication of that finding is that object motion, not object volume, is the source of visual influence on perceived heaviness.

Our goal in this study was to conceptually link the roles of visually perceived object motion and rotational inertia in heaviness perception. Our approach was inspired by studies that have demonstrated that dynamical aspects of events, such as the relative mass of colliding objects (e.g., Flynn, 1994; Gilden \& Proffitt, 1989; Runeson, 1977/1983; Todd \& Warren, 1982) or the mass of lifted objects (e.g., Bingham, 1987, 1993; Runeson \& Frykholm, 1981, 1983), can be perceived despite the fact that only motion (i.e., not forces) is visually accessible. The lawful relation between motion and dynamics inspired Runeson's kinematic specification of dynamics (KSD) principle. Kinematics is the characterization of motion in terms of displacement, velocity, and acceleration, whereas dynamics refers to masses and forces. The KSD principle underscores the fact that motion is lawfully related to the underlying dynamics that brought about the motion. Therefore, kinematic patterns of unfolding events necessarily specify the underlying dynamics of those events. Runeson suggested that optically available kinematics may, therefore, unambiguously inform a perceiver about (i.e., specify) dynamic events.

Conceptual links between kinematic information and the dynamics of wielded objects in the context of the S-W illusion have already been established. Bingham, Schmidt, and Rosenblum (1989) varied the sizes and masses of objects and asked participants to heft the objects and report which among a set of differently sized objects of the same mass could be thrown the farthest. The participants performed that perceptual task accurately.

Consistent with the S-W illusion, for an object to be optimally throwable, increases in object mass require corresponding increases in object size. Bingham et al. (1989) proposed a dynamic underpinning of this $\mathrm{S}-\mathrm{W}$ relation for throwability. Optimal throwability means dynamically that the maximum kinetic energy can be transferred to the object from the wrist. Bingham et al. demonstrated that an invariant phase lag between flexion/extension of the wrist and the elbow is exhibited for optimally throwable objects, but not for nonoptimally throwable objects (i.e., those not conforming to the $\mathrm{S}-\mathrm{W}$ relation). They proposed that information specifying optimal throwabilitythe invariant timing relation between elbow and wrist flexion/extension - is, therefore, available in the kinematics of wielding. Although the perceptual reports in Bingham et al.'s study were not of heaviness, their findings suggest that information about the dynamic properties of wielded objects is available in the kinematics of wielding.

Prior research has also demonstrated that weight can be perceived purely visually. For example, Runeson and Frykholm (1981, 1983) and Bingham $(1987,1993)$ demonstrated that the weight of objects lifted by others can be perceived solely via the kinematics of point-light displays. Those studies were conducted prior to the discovery of the inertial basis of heaviness perception; the researchers, therefore, were not in a position to make the conceptual links between inertia and kinematics proposed in the present research.

For wielded, handheld objects, motion is causally linked to the muscular forces that brought about that motion. How an object rotates in response to a given applied muscular force is dictated by the object's resistance to rotational acceleration - its rotational inertia $(I)$. For rotation in the sagittal plane, the relation between rotational force (torque; $N$ ), rotational inertia, and rotational motion is approximated by

$$
N=I \dot{\omega},
$$

where $\omega$ corresponds to rotational acceleration (Amazeen \& Turvey, 1996; Fitzpatrick, Carello, \& Turvey, 1994). ${ }^{1}$ The simplest interpretation of Equation 1 is that for a given applied torque $(N)$, rotational acceleration $(\dot{\omega})$ will increase as $I$ decreases. As was outlined above, prior research has demonstrated that heaviness perception is specific to $I$. Thus, according to Equation 1 and the KSD principle, an object that rotates faster in response to applied wielding torques specifies a lower $I$ than does an object that rotates slower in response to the same applied torques. Given that heaviness perception is specific to $I$, our conjecture is that visually detected object kinematics will influence perception of heaviness of a wielded object in a manner consistent with the inertial model. That is, if an object visually appears to rotate faster in response to a given applied torque (specifying lower $I$ ), it will be perceived as lighter than an object that visually appears to rotate slower in response to the same applied torque (specifying greater $I$ ).

In a preliminary test of the proposed hypothesis, Streit and Shockley (2005) used virtual objects (graphical depictions of wielded objects) to manipulate the apparent motion of wielded objects, relative to the objects' actual motion. Using a two-alternative forced choice method, they found that for two physically identical objects, the object whose virtual counterpart rotated $1.2 \times$ actual rotation was perceived as lighter than the virtual object that rotated $0.8 \times$ actual rotation, supporting the present hypothesis. They did not, however, control for the possibility that such cues as wrist rotation amplitude or the visual rotation amplitude of the virtual object, rather than kinematics per se, could have influenced heaviness perception. Their participants may have relied on such cues, rather than on the specifying information available in the display, since the presence of specifying information does not necessarily imply its use. For example, Gilden and Proffitt $(1989,1994)$ demonstrated that nonspecific cues, such as exit speed and scatter angle, are used to perceive the relative mass of colliding balls, rather than specifying information (i.e., relative change in velocity). Jacobs, Michaels, and Runeson (2000) and Runeson, Juslin, and Olsson (2000) demonstrated, however, that only novices used nonspecifying variables; with experience including feedback, perceivers began using specifying information. We controlled for rotation amplitude of the wrist and virtual object, using a paradigm similar to that in Streit and Shockley (2005), to rule out the possible use of those nonspecific cues. 
Because Streit and Shockley (2005) used only two alternatives, they were also unable to address whether or not perceived heaviness scaled systematically with the rotational-scaling factor. We used five levels of the rotational-scaling factor applied to the motion of the virtual objects (rotational gain), so that the virtual object would rotate more quickly (high gain) or less quickly (low gain) than the actual object. In accordance with our KSD interpretation of the inertial model of heaviness perception, we hypothesized that objects wielded in high-gain settings would be perceived as lighter than objects wielded in low-gain settings and that heaviness reports would be proportional to the magnitude of the gain manipulation.

\section{METHOD}

\section{Participants}

Thirteen undergraduate students ( 9 women, 4 men; $18-28$ years old, $M=20.6$ ) at the University of Cincinnati participated voluntarily to fulfill a course requirement. Seven of the participants were right-handed, and 6 were left-handed.

\section{Apparatus}

Wielded objects consisted of a cylindrical wooden handle $(20.5 \times$ $3 \mathrm{~cm})$ with an inserted wooden rod $(69 \times 1.25 \mathrm{~cm})$. A 100 -g fiberglass mass was placed $4 \mathrm{~cm}$ from the base of each rod, so that the mass was flush with the handle when the rod was inserted. Virtual renderings of the wielded objects were projected from an Epson PowerLite S3 projector (1,600 ANSI lumens) onto a projection screen $(117 \times 166 \mathrm{~cm})$ located $290 \mathrm{~cm}$ away from and $45^{\circ}$ to the left of the participant. A 6-d motion-tracking sensor (FasTrak II; Polhemus, Inc., Colchester, VT; sampling rate of $24 \mathrm{~Hz}$ ) was attached to the base of the object handle. The motion capture device fed data via the serial port to an IBM T40 Thinkpad (Pentium M $1.6 \mathrm{gHz}, 1 \mathrm{~GB}$ RAM), which was used to generate the virtual objects (see Figure 1). The data and rendering were managed by customized routines in MATLAB 7.0 (The MathWorks, Natick, MA).

\section{Procedure}

The participants were seated in a wooden chair and were asked to place each hand through narrow slits in an opaque occluding curtain directly in front of them. Their wrists rested on a padded wooden support on the opposite side of the curtain. One of two physically identical objects was placed in each of the participant's hands. The projection screen to the left displayed virtual depictions of each of the objects and two red spheres that were used as targets to control wielding angle amplitude (see Figure 1A; target spheres are depicted there as transparent disks for illustration purposes). One of the virtual objects (the standard object) was displayed as green, and the other (the test object) as blue; both moved in real time with the actual objects. Different colors were used for the standard and the test objects to prevent the participants from confusing the two. A scaling factor (rotational gain $=0.70,0.85,1.00,1.15$, or $1.30 \times$ actual object rotation; see Figure 1B) was applied to the virtual test object rotation (the blue virtual object). As is shown in Figure 1A, one target was always positioned at a fixed location directly above the wrist. This target dictated the resting position of both objects for periods between trials and served as one of the wielding boundaries. In order to control for the amplitude of wielding, the second target position varied, depending on the rotational amplitude condition.

The rotational amplitude manipulation controlled for the possibility that wielding amplitude (i.e., the angle subtended during wielding), rather than the responsiveness (i.e., the kinematics) of the virtual object to wielding torques, could be used as a cue. In the constant visual amplitude condition, the locations of the second target were chosen to ensure that the participants experienced the same amount of visual rotational amplitude $\left(45^{\circ}\right)$ across rotational gain changes. The resulting wrist rotation amplitudes were $58.5^{\circ}$, $51.8^{\circ}, 45.0^{\circ}, 38.3^{\circ}$, and $31.5^{\circ}$ for the five rotational gain settings, respectively. Said differently, in the constant visual amplitude condition, although visual amplitude remained constant $\left(45^{\circ}\right)$, wrist rotation covaried with rotational gain changes. In the constant wrist amplitude condition, the locations of the second target were chosen to ensure that the participants produced the same wrist rotation amplitude $\left(45^{\circ}\right)$ across rotational gain changes. The resulting visual rotation amplitudes were $31.5^{\circ}, 38.3^{\circ}, 45.0^{\circ}, 51.8^{\circ}$, and $58.5^{\circ}$ for the five rotational gain settings, respectively. In other words, in the constant wrist amplitude condition, although the wrist amplitude

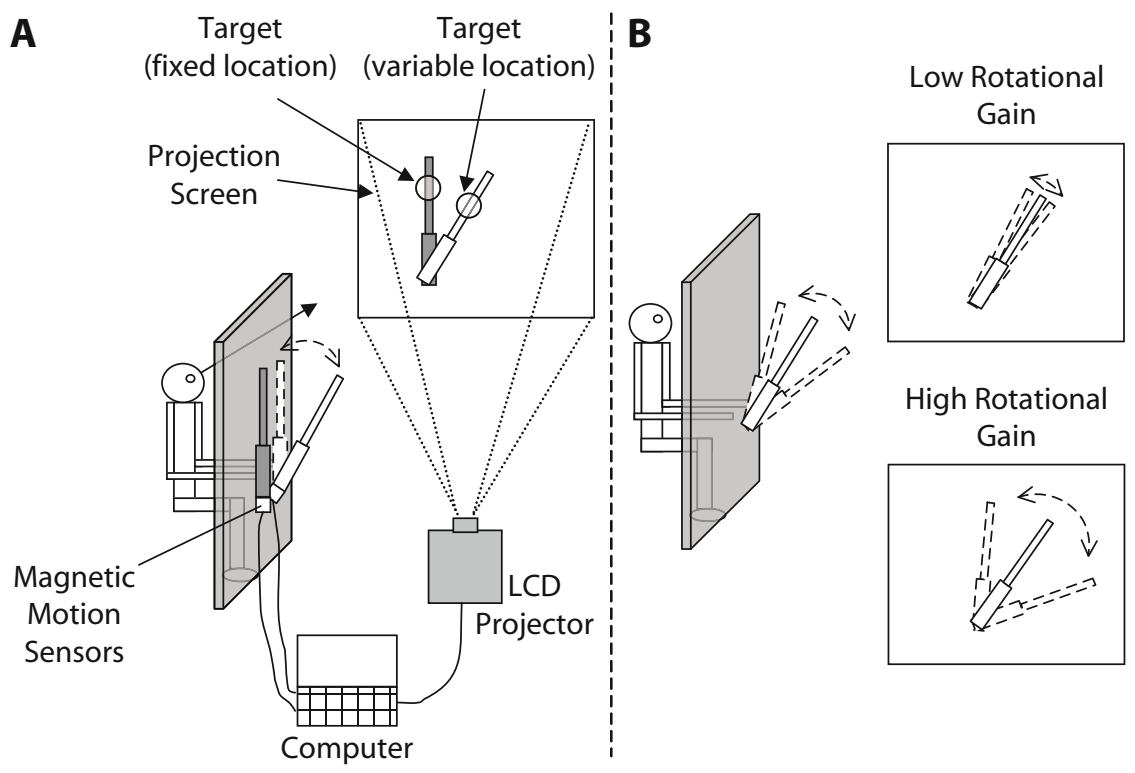

Figure 1. Illustration of method (A) and rotational gain manipulation (B). The standard object is shown in gray, and the test object in white. On the left side of panel B, the actual range of motion of the wielded object is depicted. 
remained constant $\left(45^{\circ}\right)$, visual rotation covaried with rotational gain changes.

For a given trial, a participant was instructed to rotate the standard (the green object) between the two virtual targets until he or she had an impression of its heaviness. The participant was then instructed to wield the test object (the blue object) in the same manner and could alternate between wielding the standard and the test object as many times as desired. To avoid a direct comparison of wrist and visual rotation angles, the participants were instructed to wield only one object at a time. The participant then reported how heavy the test object felt if the standard had a heaviness of 100 arbitrary units (e.g., half as heavy $=50$, twice as heavy $=200$ ). Following each report, the participant oriented both objects vertically (aligned with the fixed target), at which time the experimenter advanced the virtual display to the next trial (and accordingly, the next rotational gain setting). The experimenter then firmly grasped the test object handle (to prevent the participant from wielding it), removed the inserted wooden rod from the handle of the test object, placed it on a desk, and then reinserted the same rod back into the handle and released his hold on the handle. This procedure was used so that it would not be obvious to the participants that the same test object was used for all the trials.

One of each possible rotational gain-rotational amplitude combinations was randomly ordered within a block of 10 trials. The experiment consisted of four blocks (40 trials total) and lasted approximately $30 \mathrm{~min}$. Following completion of those trials, the participants were asked to report their impression of how many different objects had been present. The participants were then debriefed and were asked if they had realized the nature of the manipulation during the experiment. Procedures were approved by the University of Cincinnati Institutional Review Board.

\section{RESULTS}

Mean heaviness reports for each participant were submitted to a 2 (rotational amplitude) $\times 5$ (rotational gain) repeated measures ANOVA. As is illustrated in Figure 2, we found a main effect of rotational gain $[F(4,48)=$ $11.88, p<.001, \eta^{2}=1.95$ ], no main effect of rotational amplitude $\left(F<1, \eta^{2}=0.00\right)$, and no interaction between those factors $\left(F<1, \eta^{2}=0.058\right)$. The five mean heaviness reports were also submitted to a linear regression on rotational gain, which accounted for $98 \%$ of the variance in mean heaviness (regression equation: perceived heaviness $=175.46-47.89 \cdot$ rotational gain).

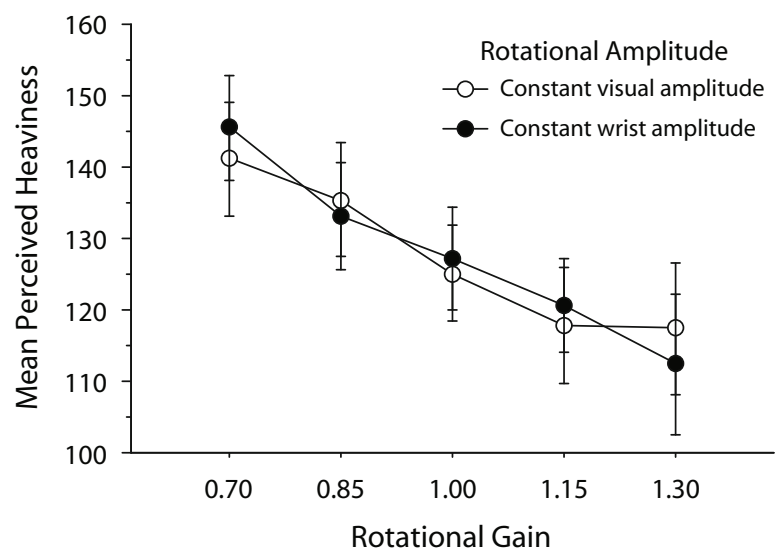

Figure 2. Mean perceived heaviness as a function of rotational gain and rotational amplitude. Error bars reflect one standard error.
The participants reported that they believed that there had been between four and six $(M=5.35, S D=1.62)$ different test objects. Only 1 participant deduced the rotational gain manipulation. When the data from that participant were excluded from analyses, the qualitative pattern of results and statistical effects did not change. All the other participants were surprised that there was only one test object.

\section{DISCUSSION}

We found that perception of heaviness was a function of the visual rotational kinematics produced in response to applied muscular torques during wielding. According to Equation 1, objects with low $I$ will rotate more in response to a given applied force than will objects with high $I$. Our results are, therefore, consistent with the inertial model of heaviness perception under our KSD interpretation. That is, if kinematics do specify the rotational inertia of a wielded object, an object that rotates less in response to a given applied torque would be expected to be perceived as heavier than an object that rotates more in response to the same applied torque.

Our results, in conjunction with prior research on heaviness perception, suggest that the rotational inertia of a wielded object can be detected both visually and haptically and that both modalities influence perceived heaviness. Visual and haptic sources of information could be perceptually independent and combine additively (cf. Amazeen, 1999; Riley \& Turvey, 2001), although it could be the case that one modality or the other dominates. Prior research has suggested, for example, that when visual and haptic proprioceptive information are discrepant, vision has the stronger influence (Hay, Pick, \& Ikeda, 1965; Pick, Warren, \& Hay, 1969). If this is the case, one would expect higher beta coefficients for an optical gain manipulation, as compared with an inertia manipulation, when both are regressed onto perceived heaviness. On the other hand, the significant visual influence observed in the present research could have resulted simply because the only variation in the stimulus was visual, and the visual influence may be weaker when presented with a concurrent inertial manipulation. Since we did not manipulate the rotational inertia of the wielded objects, the determination of the particular relation between visual and haptic information for perceived heaviness will require crossing rotational gain and inertial manipulations in future studies.

The present results are also consistent with motorcommand-expectation models of heaviness perception (Davis \& Brickett, 1977; Davis \& Roberts, 1976; Loomis, 1907; Ross \& Gregory, 1970; Woodworth, 1921). Expectation models suggest that deviations in perceived heaviness resulting from object mass manipulations reflect a discrepancy in an internal comparison of the efferent signal generated to lift an object and the afferent signal resulting from that effort. For example, with respect to the $\mathrm{S}-\mathrm{W}$ illusion, perceivers have presumably learned through experience that larger objects tend to be heavier, and so when faced with a larger object, perceivers expect it to be heavier than a smaller object. When the object moves 
more than expected in response to applied muscular force, it is perceived as lighter. Thus, according to expectation theory, objects that are visually identical with respect to size (as in the present study) would be expected to rotate the same in response to a given applied force. When an object rotates more in response to a given force than does another, expectation theory predicts that it will be perceived as lighter. Expectation theory, however, is not compatible with the results of Masin and Crestoni (1988). Masin and Crestoni found a visual influence only when objects could be seen during lifting, despite the fact that expectations of size should have arisen when the objects were viewed just prior to lifting. Furthermore, since the size of the objects in the present study never changed, the participants would have quickly recognized that object size is not informative regarding heaviness. To rule out expectation theory, however, would require an additional manipulation of the size of the virtual objects within the present paradigm. Such a manipulation would allow any influences of object size to be differentiated from influences of kinematics.

\section{AUTHOR NOTE}

This research was supported by an award by the William Seeman Fund to M.S. We also gratefully acknowledge the helpful comments of Christopher Pagano, Sverker Runeson, and one anonymous reviewer. Correspondence concerning this article should be addressed to K. Shockley, Department of Psychology, University of Cincinnati, ML 0376, 429 Dyer Hall, Cincinnati, OH 45221-0376 (e-mail: kevin.shockley@uc.edu).

\section{REFERENCES}

Amazeen, E. L. (1997). The effects of volume on perceived heaviness by dynamic touch: With and without vision. Ecological Psychology, 9, 245-263.

AmazeEn, E. L. (1999). Perceptual independence of size and weight by dynamic touch. Journal of Experimental Psychology: Human Perception \& Performance, 25, 102-119.

Amazeen, E. L., \& Turvey, M. T. (1996). Weight perception and the haptic size-weight illusion are functions of the inertia tensor. Journal of Experimental Psychology: Human Perception \& Performance, 22, 213-232.

Bingham, G. P. (1987). Kinematic form and scaling: Further investigations on the visual perception of lifted weight. Journal of Experimental Psychology: Human Perception \& Performance, 13, 155-177.

BinghaM, G. P. (1993). Scaling judgments of lifted weight: Lifter size and the role of the standard. Ecological Psychology, 5, 31-64.

Bingham, G. P., Schmidt, R. C., \& Rosenblum, L. D. (1989). Hefting for a maximum distance throw: A smart perceptual mechanism. Journal of Experimental Psychology: Human Perception \& Performance, 15, 507-528.

Charpentier, A. (1891). Analyse éxperimentale de quelques éléments de la sensation de poids [Experimental study of some aspects of weight perception]. Archives de Physiologie Normales et Pathologiques, 3, 122-135.

DAVIS, C. M., \& BRICKETT, P. (1977). The role of preparatory muscular tension in the size-weight illusion. Perception \& Psychophysics, 22, 262-264.

Davis, C. M., \& RoberTs, W. (1976). Lifting movements in the sizeweight illusion. Perception \& Psychophysics, 20, 33-36.

Ellis, R. R., \& Lederman, S. J. (1993). The role of haptic versus visual volume cues in the size-weight illusion. Perception \& Psychophysics, 53, 315-324.

FitzPatrick, P., Carello, C., \& Turvey, M. T. (1994). Eigenvalues of the inertia tensor and exteroception by the "muscular sense." Neuroscience, $\mathbf{6 0}, 551-568$.

FLYNN, S. B. (1994). The perception of relative mass in physical collisions. Ecological Psychology, 6, 185-204.

Gilden, D. L., \& Proffitt, D. R. (1989). Understanding collision dy- namics. Journal of Experimental Psychology: Human Perception \& Performance, 15, 372-383.

Gilden, D. L., \& ProffitT, D. R. (1994). Heuristic judgment of mass ratio in two-body collisions. Perception \& Psychophysics, 56, 708-720.

HAY, J. C., PICK, H. L., JR., \& IKEDA, K. (1965). Visual capture produced by prism spectacles. Psychonomic Science, 2, 215-216.

Jacobs, D. M., Michaels, C. F., \& Runeson, S. (2000). Learning to perceive the relative mass of colliding balls: The effects of ratio scaling and feedback. Perception \& Psychophysics, 62, 1332-1340.

Loomis, H. N. (1907). Reactions to equal weights of unequal size. Psychological Monographs, 8, 334-348.

Masin, S. C., \& CRestoni, L. (1988). Experimental demonstration of the sensory basis of the size-weight illusion. Perception \& Psychophysics, 44, 309-312.

PiCK, H. L., JR., Warren, D. H., \& Hay, J. C. (1969). Sensory conflict in judgments of spatial direction. Perception \& Psychophysics, 6, 203-205.

Riley, M. A., \& TuRvey, M. T. (2001). Inertial constraints on limb proprioception are independent of visual calibration. Journal of Experimental Psychology: Human Perception \& Performance, 27, 438-455.

Ross, H. E., \& GregORY, R. L. (1970). Weight illusions and weight discrimination: A revised hypothesis. Quarterly Journal of Experimental Psychology, 22, 318-328.

RUNESON, S. (1983). On visual perception of dynamic events (Acta Universitatis Upsaliensis: Studia Psychologica Upsaliensia, Serial No. 9). Uppsala: University of Uppsala. (Original work published 1977)

Runeson, S., \& Frykholm, G. (1981). Visual perception of lifted weight. Journal of Experimental Psychology: Human Perception \& Performance, 7, 733-740.

Runeson, S., \& FrYKHOLM, G. (1983). Kinematic specification of dynamics as an informational basis for person-and-action perception: Expectation, gender recognition, and deceptive intention. Journal of Experimental Psychology: General, 112, 585-615.

Runeson, S., Juslin, P., \& Olsson, H. (2000). Visual perception of dynamic properties: Cue heuristics versus direct-perceptual competence. Psychological Review, 107, 525-555.

Shockley, K., Carello, C., \& Turvey, M. T. (2004). Metamers in the haptic perception of heaviness and moveableness. Perception \& Psychophysics, 66, 731-742.

Shockley, K., Grocki, M., Carello, C., \& Turvey, M. T. (2001). Somatosensory attunement to the rigid body laws. Experimental Brain Research, 136, 133-137.

Stevens, J. C., \& Rubin, L. L. (1970). Psychophysical scales of apparent heaviness and the size-weight illusion. Perception \& Psychophysics, 8, 225-230.

Streit, M., \& SHOCKLey, K. (2005). Optical gain and the perception of heaviness. In H. Heft \& K. L. Marsh (Eds.), Studies in perception and action VIII (pp. 185-187). Mahwah, NJ: Erlbaum.

ToDD, J. T., \& WARREN, W. H., JR. (1982). Visual perception of relative mass in dynamic events. Perception, 11, 325-335.

Turvey, M. T., Shockley, K., \& Carello, C. (1999). Affordance, proper function, and the physical basis of perceived heaviness. Cognition, 73, B17-B26.

Weber, E. H. (1978). The sense of touch (H. E. Ross, Ed. and Trans.). London: Academic Press. (Original work published 1834)

Woodworth, R. S. (1921). Psychology: A study of mental life. New York: Holt.

\section{NOTE}

1. A complete characterization of the relation between torque $(N)$, rotational inertia $(I)$, and rotational acceleration $(\dot{\omega})$ is captured by

$$
N_{1}=I_{1} \dot{\omega}_{1}-\omega_{2} \omega_{3}\left(I_{2}-I_{3}\right),
$$

where $\omega$ corresponds to angular velocity and subscripts 1,2 , and 3 correspond to the three spatially orthogonal symmetry axes (eigenvectors) axes dictated by the mass distribution about a given point of rotation so that masses are evenly distributed about those axes. Rotation in the sagittal plane approximates rotation about Axis 1 . In that case, $\omega_{2}$ and $\omega_{3}$ approach zero, effectively reducing Equation 2 to Equation 1.

(Manuscript received January 8, 2006; revision accepted for publication March 15, 2006.) 